\title{
Initial Growth Mode of Au on Ag(110) Studied with First-Principles Calculations
}

\author{
C. T. Chan, ${ }^{(1)}$ K. P. Bohnen, ${ }^{(2)}$ and K. M. Ho ${ }^{(1),(3)}$ \\ (1) Ames Laboratory-U.S. DOE, Iowa State University, Ames, Iowa 50011 \\ ${ }^{(2)}$ Kernforschungszentrum Karlsruhe, Institut für Nukleare Festkörperphysik, Karlsruhe, Federal Repubiic of Germany \\ ${ }^{(3)}$ Department of Physics, Iowa State University, Ames, Iowa 50011
}

(Received 4 May 1992)

\begin{abstract}
We studied the initial growth mode of $\mathrm{Au}$ on $\mathrm{Ag}(110)$ using first-principles total energy calculations. We found that a recently observed bilayer growth mode in this system is not energetically favorable and thus may not be an equilibrium process. The most favorable initial growth process up to 1 monolayer $\mathrm{Au}$ coverage is found to proceed via subsurface substitution, which is an interesting growth mode for a metal-on-metal system.
\end{abstract}

PACS numbers: $68.35 . \mathrm{Bs}, 68.35 . \mathrm{Md}$

The growth of one material on a substrate of another material can be classified into three distinct growth modes: layer by layer, 3D crystallites formation, and layer-by-layer growth followed by $3 \mathrm{D}$ crystallites formations. These growth modes are known as the Frank-van der Merwe (FM), Volmer-Weber (VW), and StranskiKrastanov (SK) modes, respectively [1], and most growth processes belong to either one of these three categories. In principle, the growth mode can be predicted, or at least discussed using Bauer's criteria [1], if we know the surface free energy of the substrate $\left(\gamma_{s}\right)$ and that of the overlayer $\left(\gamma_{o}\right)$, as well as the interfacial interaction energy $\left(\gamma_{i}\right)$. However, the interface energy $\gamma_{i}$ is usually an unknown quantity (an order-of-magnitude estimate can sometimes be obtained from bulk alloy heat of formation or grain-boundary data). Even the values of $\gamma_{s}$ and $\gamma_{o}$ cannot be determined accurately since experimentally available surface tension data are usually obtained for surfaces with mixed rather than specific surface orientations, and usually measured at elevated temperatures. Moreover, the overlayer seldom has the same lattice constant as the substrate, and the surface free energy of a strained lattice cannot be estimated easily. For ultrathin layers, the separation of the surface energies into its individual components is also nonunique, and its validity and transferability depends on whether a microscopic model can be used to describe the interface. To complicate matters further, there are growth processes that are notable exceptions to the three broad categories that we have mentioned above, as observed by several recent experiments [2]. Even very simple systems, such as the Au on $\mathrm{Ag}(110)$ system we are addressing in this paper, can have unexpected growth phenomena as we will show below. A quantitative study of growth processes is thus a formidable problem for theorists. It has been remarked that a truly microscopic treatment for growth models is yet another challenge for the future of surface physics [3], but on the other hand, a truly microscopic treatment may well be the only reliable way to study these phenomena theoretically.

Recently, Fenter and Gustafsson [4,5] studied the growth of $\mathrm{Au}$ on $\mathrm{Ag}(110)$, using medium-energy ion scattering. Au and $\mathrm{Ag}$ have almost the same lattice constants (4.08 and $4.09 \AA$, respectively), comparable surface energies ( $\mathrm{Ag}$ is lower), and similar electronic and structural properties because they are isoelectronic. $\mathrm{Au}$ is thus expected to follow a simple epitaxial growth mode on Ag, but Fenter and Gustafsson [4,5] observed a rather unconventional pattern of growth when they deposited $\mathrm{Au}$ on $\mathrm{Ag}(110)$. They observed that in the initial growth process, half of the Au atoms occupy second-layer sites; there is no significant penetration of $\mathrm{Au}$ into the Ag bulk; and only about half of the Ag surface is covered after 1 monolayer (ML) of $\mathrm{Au}$ is deposited. There is also significant $\mathrm{Ag}$ yield even at 2 or more ML of $\mathrm{Au}$. After a quantitative data analysis [4], the experimental observation was interpreted to be a bilayer growth of $\mathrm{Au}$, which was quite unusual for a metal-on-metal system.

For a metal-on-metal system, especially for $\mathrm{Ag}$ and $\mathrm{Au}$ that are relatively "spherical" and have no tendency for directional bonding, a bilayer growth is very interesting and warrants a study via first-principles calculations. The case of $\mathrm{Au} / \mathrm{Ag}(110)$ is also a nice prototypical system for the study of growth because this system is relatively simple and thus tractable with first-principles calculations, and yet the system exhibits interesting growth behavior. For computation purposes, it is important that interesting physics are observed with very thin layers, so that we do not need to deal with too many atomic degrees of freedom and do not have to worry about extra complications like misfit dislocations.

The calculations were done within the framework of local-density-functional formalism [6]. We used the Hedin-Lundqvist [7] form of exchange-correlation functional and norm-conserving pseudopotentials [8] for the $\mathrm{Au}$ and $\mathrm{Ag}$ ionic cores. The Bloch wave functions are expanded in a mixed basis [9] of plane waves (kinetic energy up to $12 \mathrm{Ry}$ ) and numerical functions centered on atomic sites. The same method has been applied successfully to many transition and noble-metal systems including $\mathrm{Au}$ and $\mathrm{Ag}$. The composite $\mathrm{Ag}-\mathrm{Au}$ system is represented by repeated slabs with thickness up to 11 layers, 
separated by a vacuum equivalent in thickness to 5 layers. Since surface alloying situations are considered, a $c(2 \times 2)$ unit cell is employed. The $k$ points are sampled on a uniform grid of 35 points in the irreducible Brillouin zone. The atomic positions for the Au overlayers and the Ag substrate are determined by relaxing the atoms according to the Hellmann-Feynman forces [10]. In some of the mixed-layer configurations we have considered, buckling of the atomic layers has to be taken into account because $\mathrm{Au}$ and $\mathrm{Ag}$ atoms in the same layer are not required by symmetry to have the same vertical positions.

By considering the surface energy change as we put up to 2 pseudomorphic layers of $\mathrm{Au}$ on $\mathrm{Ag}(110)$, we have concluded that a bilayer growth mode is not energetically favorable [11]. This notion can be reinforced by considering the heat of formation per atom in the growth process, which we define operationally as the energy gained when a $\mathrm{Au}$ atom that originates from a bulk $\mathrm{Au}$ environment is incorporated into the Ag substrate (the bigger this number, the stronger the bonding; and a negative number would indicate that $\mathrm{Au}$ does not wet $\mathrm{Ag}$ ) [12]. The heat of formation for the layer-by-layer case is listed in the second column of Table I. We see from Table I that the $0.05-\mathrm{eV} /$ atom heat of formation corresponding to a $\mathrm{Au}$ bilayer on top of the $\mathrm{Ag}(110)$ substrate is substantially less than the $0.12 \mathrm{eV} /$ atom when a single Au layer covers Ag, so there is no reason why a bilayer growth can "preempt" a single layer growth. In fact, since the heat of formation for a Au bilayer is less than half of that of a monolayer, the incoming Au atoms would be better off in energy to be in a bulk environment (forming crystallites) than covering the surface after the first Au monolayer is deposited. So, within the realm of the three traditional growth modes, Au should grow on $\mathrm{Ag}$ (110) according to the SK mode. The next scenario we considered is the case of intermixing (surface alloying) where half the $\mathrm{Ag}$ sites on the top layers are substituted by Au. Au and Ag form bulk alloys, and the possibility of intermixing has also been considered as an alternative model that can explain the experimentally observed behavior, although the bilayer model was favored since it provides better quantitative fits to the data with fewer fitting parameters and

TABLE I. The heats of formation for various coverages of $\mathrm{Au}$ on $\mathrm{Ag}(110)$. Both layer-by-layer growth and ordered surface alloy formation are considered. The numbers in the parentheses indicate the number of $\mathrm{Au}$ or $\mathrm{Au} / \mathrm{Ag}$ mixed layers on one side of the slab.

\begin{tabular}{ccc}
\hline \hline $\begin{array}{c}\text { Au coverage } \\
(\mathrm{ML})\end{array}$ & Alloy & $\begin{array}{c}\text { Heat of formation } \\
(\mathrm{eV} / \text { atom) } \\
\text { Layer by layer }\end{array}$ \\
\hline 0.5 & $0.189(1)$ & \\
1.0 & $0.182(2)$ & $0.122(1)$ \\
1.5 & $0.174(3)$ & \\
2.0 & $0.175(4)$ & $0.050(2)$ \\
\hline \hline
\end{tabular}

less unusual features [5]. We can only consider the case of ordered surface alloys within the present first-principles formulation. The heats of formation per Au atom for various geometries up to $2 \mathrm{ML}$ (4 alloy layers) are listed in the first column of Table I, where we can see that the binding is overwhelmingly stronger in forming $\mathrm{Au}$-Ag intermixed layers than a layer-by-layer overlayer formation at all the coverages we have considered. Unless the $\mathrm{Au}$ atoms cannot penetrate the top Ag layer due to kinetic barriers, a surface alloy should be formed in favor of layer-by-layer growth.

Given the fact that a mixed-layer configuration is energetically more favorable than layer-by-layer or bilayer formations, it is tempting to conclude that this is what actually has happened in the experiment; especially the experimental observation does not rule out surface alloying. However, there is another possibility that we cannot overlook. Ag has a lower surface energy than $\mathrm{Au}$. Is it possible that the $\mathrm{Au}$ overlayers are reverse-coated by 1 or more layers of $\mathrm{Ag}$ ? To address this issue, we considered a few sandwich configurations in which either a Au layer or a Au-Ag mixed layer is embedded under 1 or more layers of Ag. The case for a pure Au layer (1-ML Au coverage) is shown in Fig. 1, where we plot the heat of formation per $\mathrm{Au}$ atom for the $\mathrm{Au}$ layer (i) at the top, (ii) covered by 1 layer of $\mathrm{Ag}$, and (iii) covered by 2 layers of Ag. As can be seen from Fig. 1, the Au layer obviously has the highest binding energy when it is covered by 1 layer of $\mathrm{Ag}$. The case for a mixed $\mathrm{Au}-\mathrm{Ag}$ layer $(0.5-\mathrm{ML}$ Au coverage) is presented in Fig. 2, where we plot the heat of formation per $\mathrm{Au}$ atom for the mixed layer being inserted at various positions. It is clear from Fig. 2 the most favorable configuration is also under 1 layer of $\mathrm{Ag}$. Hence, whether it is a complete layer of $\mathrm{Au}$ or a mixed $\mathrm{Au}-\mathrm{Ag}$ alloy layer, the energy is most favorable when the $\mathrm{Au}$ atoms are close to the surface and coated by 1 layer

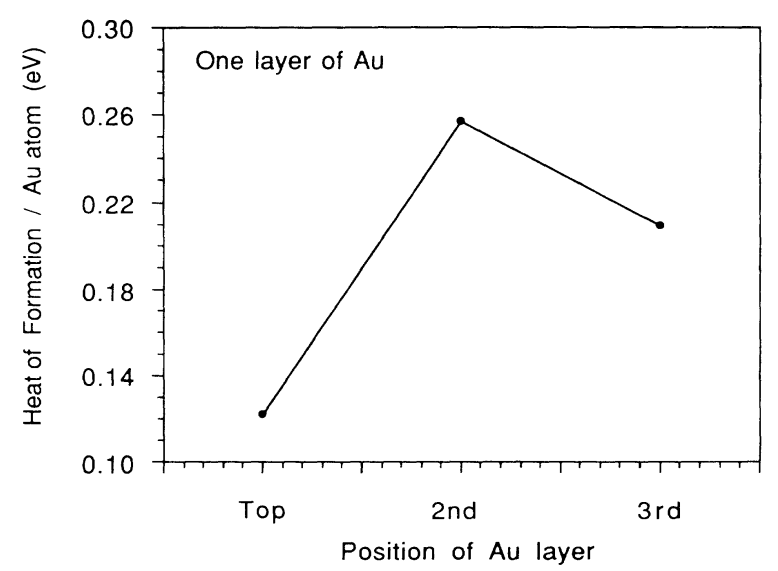

FIG. 1. Heats of formation per Au atom for 1 layer of $\mathrm{Au}$, with the $\mathrm{Au}$ layer at various positions in the $\mathrm{Au} / \mathrm{Ag}(110)$ system. Note that the energy is most favorable when the $A u$ is covered by 1 layer of $\mathrm{Ag}$. 


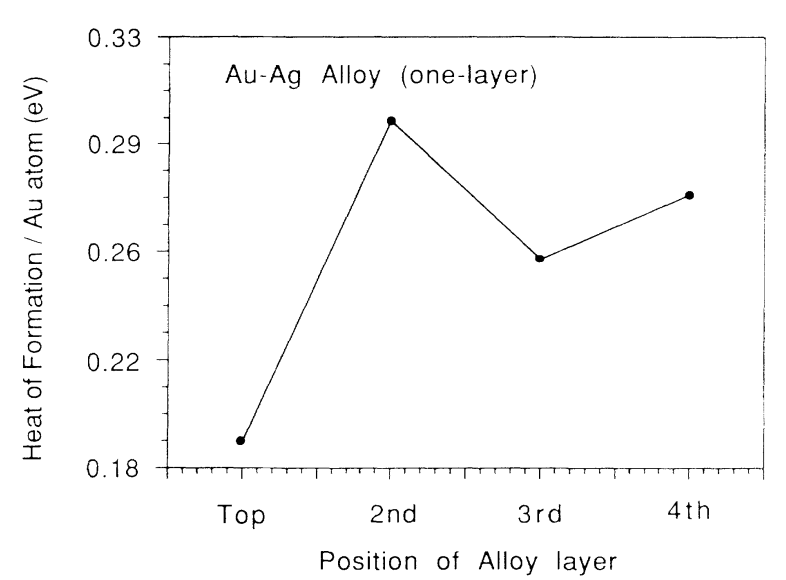

FIG. 2. Heats of formation per Au atom for a Au-Ag alloy layer at various positions in the $\mathrm{Au} / \mathrm{Ag}(110)$ system. The energy is also most favorable when the layer is covered by 1 layer of $\mathrm{Ag}$.

of $\mathrm{Ag}$. The heat of formation for $\mathrm{Au}$ in the $0.5-\mathrm{ML}$ mixed-layer configuration is higher than that in the pure Au layer ( $1 \mathrm{ML}$ ) configuration, implying that during the initial growth process when the Au coverage is submonolayer, the $\mathrm{Au}$ atoms would tend to distribute themselves evenly at the subsurface sites (in the second layer under 1 layer of $\mathrm{Ag}$ ) in the initial growth. Entropy effects will also favor an evenly distributed arrangement. We also found that at $1-\mathrm{ML}$ coverage, the configuration with 1 layer of $\mathrm{Au}$ under 1 layer of $\mathrm{Ag}$ has a heat of formation of about $0.26 \mathrm{eV}$ per $\mathrm{Au}$ atom, which is higher than with 2 layers of $\mathrm{Au}-\mathrm{Ag}$ alloy with the same number of $\mathrm{Au}$ atoms, irrespective of whether the alloy layers are covered by a layer of $\mathrm{Ag}$ or not. Thus, all the Au atoms should be found in the second layer until all the Ag atoms are replaced. The present calculations do not give enough information to judge conclusively what should happen for higher coverages.

In summary, we have studied the initial growth mode of $\mathrm{Au}$ on $\mathrm{Ag}(110)$ with first-principles calculations. We found that a bilayer growth of Au is very unfavorable in energy and surface-alloy formation is much better in energy. However, the energetically most favorable initial growth mode (up to $1 \mathrm{ML}$ ) proceeds with the Au atoms substituting Ag atoms in the second layer. We note that the (110) surface of a fcc metal is quite an open surface, and the second layer is not shadowed by the top layer atoms. A subsurface substitution of the Ag atoms by $\mathrm{Au}$ is thus quite plausible as long as the energy is favorable. Our initial motivation is to understand a novel bilayer metal-on-metal growth mode that was reported in medium-energy-ion scattering experiments by Fenter and Gustafsson [4,5], and we end up encountering another interesting growth mode. Our results are not consistent with the interpretation of the medium-energy-ionscattering experiment $[4,5]$. We should emphasize that, with first-principles total energy calculations, we have considered the energetics of the growth process, and the conclusion is relevant to equilibrium situations. The bilayer mode is observed by depositing $\mathrm{Au}$ on $\mathrm{Ag}$ at room temperature without further annealing, and thus might be a nonequilibrium process where the kinetics may dominate over the energetics $[5,13]$. In this case, our theoretical results and the bilayer interpretation of the experiment are not mutually exclusive. On the other hand, Rousset et al. [14] have very recently studied the same system under similar physical conditions with scanning tunneling microscopy and also reexamined the mediumenergy-ion-scattering data of Fenter and Gustafsson. They came to the conclusion that in submonolayer $\mathrm{Au}$ coverages, the $\mathrm{Ag} / \mathrm{Au}$ forms an intermixed layer with practically all of the $\mathrm{Au}$ atoms in the second layer, located below a top layer of $\mathrm{Ag}$. This is entirely consistent with our theoretical result. There is thus still some controversy over the interpretation of the same piece of data, or what actually has happened during the deposition process at room temperature, and probably more experiments are needed to settle the issue. However, the present calculation should set the stage for what should be expected when the system is in equilibrium. We also note that there is at least one other metal/metal system that grows via a subsurface mode in a sandwich configuration [15].

This calculation shows that a truly microscopic treatment of growth is possible within the framework of firstprinciples total energy calculations. The energy and forces for many atomic arrangements as computed from first principles are available as a by-product, which could serve as a data base for fitting to semiempirical interatomic potentials that would allow us to extrapolate our results to cover more complex phenomena such as the kinetics of growth. This is particularly useful since information for interatomic potentials between different atomic species is not so easy to obtain.

We thank Dr. P. Fenter and Professor T. Gustafsson for discussions and Dr. S. Chiang for sending us a preprint of her work. Ames Laboratory is operated for the U.S. Department of Energy by Iowa State University under Contract No. W-7405-ENG-82. This work is supported by the Director of Energy Research, Office of Basic Energy Sciences, including a grant of computer time on the Cray computer at the Lawrence Livermore Laboratory and NATO collaborative Grant No. RG(86/0516). Warm hospitality at INFP-Kernforschungszentrum Karlsruhe is gratefully acknowledged by one of us (C.T.C.).

[1] See, e.g., E. Bauer, Appl. Surf. Sci. 11/12, 479 (1982).

[2] See, e.g., B. J. Hinch, C. Koziol, J. P. Toennies, and G. Zhang, Europhys. Lett. 10, 341 (1989); H. Li and B. P. 
Tonner, Surf. Sci. 237, 141 (1990); Y. Zhang and A. J. Slavin, J. Vac. Sci. Technol. A 9, 1784 (1991).

[3] A. Zangwill, Physics at Surfaces (Cambridge Univ. Press, Cambridge, 1988), p. 432.

[4] P. Fenter and T. Gustafsson, Phys. Rev. Lett. 64, 1142 (1990).

[5] P. Fenter and T. Gustafsson, Phys. Rev. B 43, 12195 (1991).

[6] See, e.g., Theory of the Inhomogeneous Electron Gas, edited by N. H. March and S. Lundqvist (Plenum, New York, 1983).

[7] L. Hedin and B. I. Lundqvist, J. Phys. C 4, 2064 (1971).

[8] D. R. Hamann, M. Schluter, and C. Chiang, Phys. Rev. Lett. 43, 1494 (1979); G. B. Bachelet and M. Schluter, Phys. Rev. B 25, 2103 (1982).

[9] S. G. Louie, K. M. Ho, and M. L. Cohen, Phys. Rev. B 19, 1774 (1979).

[10] C. Elsaesser, K. M. Ho, C. T. Chan, and M. Fahnle, J.
Phys. Condens. Matter (to be published).

[11] K. P. Bohnen, C. T. Chan, and K. M. Ho, Surf. Sci. (to be published).

[12] We could also, if we like, consider the change in "surface energy," with the surface energy operationally defined as the difference between the total energy of the slab and the energy of the individual atoms in their respective bulk environment. The conclusion will be the same. In those cases where the different configurations have the same stoichiometry, we can simply compare the total energy of the slabs, but considering the heat of formation (as defined here) would allow us to compare and discuss more easily configurations that have different Au coverages.

[13] P. Fenter (private communication).

[14] S. Rousset, S. Chiang, D. E. Fowler, and D. D. Chambliss (to be published).

[15] P. J. Schmitz, W. Y. Leung, G. W. Graham, and P. A. Thiel, Phys. Rev. B 40, 11477 (1989). 